\title{
Field-Direction Dependence of the Upper Critical Field in Organic Superconductors
}

\author{
M.D. Croitoru • M. Houzet · A.I. Buzdin
}

\begin{abstract}
In this work, we investigate the anisotropy of the in-plane critical field in conventional and spatially modulated phases of layered superconductors within the quasi-classical approach, taking into account the interlayer Josephson coupling. We show that the anisotropy of the onset of superconductivity may change dramatically in the FFLO state as compared with the conventional superconducting phase.
\end{abstract}

Keywords FFLO state $\cdot$ Anisotropy $\cdot$ Critical field

In the last decades, the quasi low-dimensional correlated electron systems have been the focus of the theoretical and experimental investigations due to their rich variety of normal and superconducting properties not found in threedimensional materials [1-8]. Amongst them, layered superconductors subjected to an external magnetic field have attracted much attention $[1,9]$. Indeed, in organic layered superconductors, (TMTSF) $)_{2} \mathrm{X}$, where anion $\mathrm{X}$ is $\mathrm{PF}_{6}, \mathrm{ClO}_{4}$, etc., a very large upper critical fields, exceeding the Pauli paramagnetic limit, for a magnetic field aligned parallel to their conducting layers were reported [10-14]. The reason is that, in layered conductors, the spatial orbital motion of electrons is mostly restricted to the conducting layers and the orbital depairing due to the in-plane magnetic field is strongly quenched. Hence, the magnetic field only influences spins

M.D. Croitoru $(\varangle)$ · A.I. Buzdin

Université Bordeaux I, LOMA, UMR 5798, 33405 Talence,

France

e-mail: mikhail.croitoru@uni-bayreuth.de

M. Houzet

DRFMC/SPSMS/LCP, CEA, Grenoble, 38054 Grenoble Cedex 9,

France and singlet pairing is mainly limited by the Zeeman effect. Nevertheless, this limitation can be surmounted by allowing a spin-triplet state or a spatially modulated spin-singletstate (limit is upshifted) $[15,16]$. Whether a spin part of the superconducting order parameter singlet or triplet is currently under discussion because the NMR experiment with (TMTSF) ${ }_{2} \mathrm{PF}_{6}$ salt at $T_{c}$ and under pressure showed the absence of the Knight shift, supporting the triplet scenario of pairing [17], while the ${ }^{77} \mathrm{Se}$ NMR Knight shift in recent experiment with (TMTSF) ${ }_{2} \mathrm{ClO}_{4}$ reveals a decrease in spin susceptibility $\chi_{s}$ consistent with singlet spin pairing [18]. ${ }^{13} \mathrm{C}$ NMR measurements with $\kappa$-(BEDT-TTF) ${ }_{2} \mathrm{Cu}(\mathrm{NCS})_{4}$ evidenced for a Zeeman-driven transition within the superconducting state and stabilization of inhomogeneous, spatially modulated phase [19].

Recently the field-amplitude and field-angle dependence of the superconducting transition temperature $T_{c}(H)$ of the organic superconductor (TMTSF) ${ }_{2} \mathrm{ClO}_{4}$ in magnetic field applied along the conduction planes have been reported [14]. The authors observed an upturn of the curve of the upper critical field at low temperatures. This behavior has often been discussed in connection with the possibility of the FFLO state formation [20-23]. Moreover, an unusual inplane anisotropy of $H_{c 2}$ in the high-field regime was observed, which authors interpret as an evidence of the FFLO state formation. Motivated by the experimental findings, in this work we investigate the influence of the modulating superconducting phase on the in-plane anisotropy of $H_{c 2}$ in layered conductors.

We consider here a quasi-two-dimensional superconductor in magnetic field applied parallel to the plane of the most conductive layers ( $x y$-plane). The system single-electron spectrum is approximated by $\xi_{p}=E_{p}^{(2)}+\varepsilon\left(p_{z}\right)$, where $E_{p}^{(2)}=\frac{\widehat{p}_{x}}{2 m_{x}}+\frac{\widehat{p}_{y}}{2 m_{y}}$, and the tight-binding approximation is used to describe the electron motion along the $z$-direction: 
$\varepsilon\left(p_{z}\right)=2 t \cos \left(p_{z} d\right)$ [24]. The coupling between layers, $t$, satisfies the condition $\frac{T_{c 0}^{2}}{E_{F}} \ll t \ll T_{c 0}$. Here $T_{c 0}$ is the critical temperature of the system at $H=0$ with neglected interlayer coupling and $E_{F}$ is the Fermi energy. The Eilenberger equation describing such systems acquires the form [25]

$\widehat{L}_{2} f_{\omega}\left(\mathbf{n}_{\mathbf{p}}, \mathbf{r}, p_{z}\right)=\Delta g_{\omega}\left(\mathbf{n}_{\mathbf{p}}, \mathbf{r}, p_{z}\right)$,

where $g_{\omega}$ and $f_{\omega}$ are the quasiclassical Green functions, operator $\widehat{L}_{2}=\Omega_{n}+\frac{1}{2} \mathbf{v}_{F} \nabla_{x, y}+t \sin \left(p_{z} d\right)\left[e^{i \mathbf{Q r}}-e^{-i \mathbf{Q r}}\right]$ with $\Omega_{n} \equiv \omega_{n}-i h$ and $\mathbf{Q}=\frac{\pi d}{\phi_{0}}\left(-H_{y}, H_{x}\right)$ with $\phi_{0}=\pi \hbar c / e$. Here $h \equiv \mu_{B} H$ is the Zeeman energy, $\mathbf{v}_{F}$ is the 2D Fermi velocity and $d$ is the interlayer distance. In the following, we assume that the system is near the transition, therefore $g_{\omega}\left(\mathbf{n}_{\mathbf{p}}, \mathbf{r}, p_{z}\right) \simeq \operatorname{sign}\left(\omega_{n}\right)$, and that the mean free path inside the layers are much larger than the corresponding coherence length.

Restricting ourselves first to the case when paramagnetic limit (FFLO state) is absent, performing averaging over the Fermi surface, and using the iterative procedure with respect to $f_{\omega}\left(\mathbf{n}_{\mathbf{p}}, \mathbf{r}, p_{z}\right)$ we obtain the extended LowerenceDoniach equation (in the sequel, MLD equation)

$\Delta(\mathbf{r}) \ln \left(\frac{T}{T_{c 0}}\right)=\pi T \sum_{n} \widehat{L}_{\mathrm{MLD}}\left(\omega_{n}\right) \Delta(\mathbf{r})$

with $\widehat{L}_{\mathrm{MLD}}\left(\omega_{n}\right) \equiv 1 /\left(4 \omega_{n}^{3}\right)\left\{\left\langle v_{F x}^{2}\right\rangle \partial_{x}^{2}+\left\langle v_{F y}^{2}\right\rangle \partial_{y}^{2}\right\}-$ $t^{2}[1-\cos (2 \mathbf{Q r})] / \omega_{n}^{3}+\left[\left\langle v_{F x}^{2}\right\rangle Q_{x}^{2}+\left\langle v_{F y}^{2}\right\rangle Q_{y}^{2}\right] \times t^{2}[1-$ $7 \cos (2 \mathbf{Q} \mathbf{r})] / 4 \omega_{n}^{5}$. In order to obtain the iterative scheme convergent, we have required $\Delta(\mathbf{r}) \gg \mathbf{v}_{F} \nabla_{x, y} \Delta(\mathbf{r}) / 2 \pi T_{c 0}$, which implies that the characteristic scale of the order parameter variations should be much smaller than $\xi_{0}=$ $v_{F} / 2 \pi T_{c 0}$. The MLD equation contains the term proportional to $\left(v_{F} Q\right)^{2} t^{2}$, which is absent in the LowerenceDoniach equation. In the case of $v_{F} Q \ll T_{c 0}$, one may neglect the last term and obtain the standard LowerenceDoniach equation.

The angle resolved highest magnetic field at which superconductivity can nucleate in a sample is obtained as

$\left.H_{c 2}(\alpha, T)\right|_{\lim }=\frac{\left.H_{c 2}\left(\frac{\pi}{2}\right)\right|_{\lim }}{\sqrt{\sin ^{2}(\alpha)+\frac{m_{x}}{m_{y}} \cos ^{2}(\alpha)}}$,

where $\alpha$ is the angle between the direction of the external field and $x$-axis. Here $\left.H_{c 2}\left(\frac{\pi}{2}\right)\right|_{\lim =\varkappa_{I}}=\frac{m_{x} v_{F} T_{c 0}}{\hbar d t} \frac{\phi_{0}}{2 \pi}(1-$ $\left.\frac{T}{T_{c 0}}\right)$, when $H \ll \frac{t}{\pi d v_{F}} \phi_{0}\left(\varkappa_{I}\right)$. The MLD equation in the regime of magnetic fields, $t \phi_{0} / \pi d v_{F} \ll H \ll \sqrt{t T_{c 0}} \phi_{0} /$ $\pi d v_{F}\left(\varkappa_{I I}\right)$ gives rise to $\left.H_{c 2}\left(\frac{\pi}{2}\right)\right|_{\varkappa_{I I}}=\frac{7 \zeta(3) t^{2}}{8 \pi^{3} d T_{c 0}} \sqrt{\frac{m_{x}}{T-T_{c t}}} \phi_{0}$. In the regime $\varkappa_{I I I}$ of the magnetic fields, $\frac{\phi_{0}}{\pi d v_{F}} \sqrt{t T_{c 0}} \ll H \ll$ $\frac{\phi_{0}}{\pi d v_{F}} T_{c 0}$, the MLD equation describes the beginning of the reentrant superconductivity regime [26, 27] with the upper critical field defined as

$\left.H_{c 2}\left(\frac{\pi}{2}\right)\right|_{\varkappa_{I I I}}=\sqrt{\frac{28}{31} \frac{\zeta(3)}{\zeta(5)}} \frac{T_{c t} \sqrt{T-T_{c t}}}{\hbar d t} \frac{\sqrt{2 m_{x}}}{\hbar} \phi_{0}$.
Here, one can see that $T>T_{c t}$, where $T_{c t}$ is introduced via the relation $\ln \left(T_{c 0} / T_{c t}\right)=\frac{7 \zeta(3)}{4 \pi^{2}} \frac{t^{2}}{T_{c 0}^{2}}$.

The previous treatment can be extended to extremely large magnetic fields, provided that one can omit the assumption that the characteristic scale of the order parameter variations is much smaller than $\xi_{0}$. For this purpose, we need to reconsider the solution of the Eilenberger equation. According to the 2D Eilenberger equation, the magnetic field induced potential has the form $V(\mathbf{r})=2 i t \sin \left(p_{z} d\right) \sin (\mathbf{Q r})$, i.e., it is periodic in real space. Since the system under consideration is in an arbitrary magnetic field, the solution of Eq. (1) can be written without any loss of generality as a Bloch function

$f_{\omega}\left(\mathbf{n}_{\mathbf{p}}, \mathbf{r}, p_{z}\right)=e^{i \mathbf{q r}} \sum_{m} e^{i m \mathbf{Q r}} f_{m}\left(\omega_{n}, \mathbf{n}_{\mathbf{p}}, p_{z}\right)$,

or $f_{\omega}\left(\mathbf{n}_{\mathbf{p}}, \mathbf{r}, p_{z}\right)=e^{i \mathbf{q r}} \widetilde{f}_{\omega}\left(\mathbf{n}_{\mathbf{p}}, \mathbf{r}, p_{z}\right)$, where $\widetilde{f}_{\omega}\left(\mathbf{n}_{\mathbf{p}}, \mathbf{r}, p_{z}\right)$ has the periodicity of $2 \pi / \mathbf{Q}$. In Eq. (5), we take into account the possibility for the formation of the pairing state $\left(\mathbf{k}+\frac{\mathbf{q}}{2} \uparrow\right.$; $\left.-\mathbf{k}+\frac{\mathbf{q}}{2} \downarrow\right)$ with the modulation vector $q \sim 2 \mu_{B} H / \hbar v_{F}$. Because of the form for $f_{\omega}\left(\mathbf{n}_{\mathbf{p}}, \mathbf{r}, p_{z}\right)$ of Eq. (5), one can write $\Delta(\mathbf{r})$ as

$\Delta(\mathbf{r})=e^{i \mathbf{q r}} \sum_{m} e^{i 2 m \mathbf{Q r}} \Delta_{2 m}$.

From symmetry considerations, it follows that $\Delta_{-2 m}=\Delta_{2 m}$. Substituting Eqs. (5) and (6) into Eq. (1) and retaining in the sum only the zero and the first term, since we want to investigate the regime $H \gg \frac{T_{c 0}}{\pi d v_{F}} \phi_{0}\left(T_{c 0} \ll v_{F} Q\right)$, when $q=0$ one gets after averaging over the Fermi surface

$\ln \left(\frac{T_{c 0}}{T}\right)=\pi T \sum_{n} \frac{t^{2}}{\omega_{n}^{2}}\left[\omega_{n}^{2}+\left(\frac{v_{F} Q}{2}\right)^{2}\right]^{-1 / 2}$.

For extremely large values of the external magnetic field, we can use $\omega_{n} \sim T_{c 0} \ll v_{F} Q$ and obtain Eq. (3) with $\left.H_{c 2}\left(\frac{\pi}{2}\right)\right|_{\varkappa_{I V}}=\frac{t^{2}}{2 d v_{F}} \frac{\phi_{0}}{\left(T_{c 0}-T\right)}$. Therefore, summarizing the above discussion, we can conclude that in the non-modulated phase the in-plane anisotropy of the upper critical magnetic field can be defined by Eq. (3) and $\left.H_{c 2}\left(\frac{\pi}{2}\right)\right|_{\text {lim }}$ should be taken accordingly, as described in Table 1.

As it is known $[23,28]$, the FFLO state only appears at $T<T^{*} \simeq 0.56 T_{c 0}$ or $H>H^{*} \simeq 1.06 T_{c 0} / \mu_{B}$. Therefore, $v_{F} Q \gtrsim T_{c 0}$ or $\sqrt{t T_{c 0}} \ll\left(v_{F} Q\right)$. This allows to retain only the zero and first term in Eq. (5). Expanding up to the second order in $t / T_{c 0}$ and making use of the average scheme for the Fermi surface as, $\langle\ldots\rangle \equiv \int_{-\pi / d}^{\pi / d} \frac{d d p_{z}}{2 \pi} \int_{0}^{2 \pi} d \varphi(\ldots)$, one obtains [25]

$$
\begin{aligned}
\ln \left(\frac{T_{c 0}}{T}\right) \\
=\pi T \sum_{n}\left\{\frac{1}{\omega_{n}}-\left\langle\frac{1}{L_{n}(\mathbf{q})}\right\rangle\right. \\
\left.\quad+\frac{t^{2}}{2}\left\langle\frac{1}{L_{n}(\mathbf{q}+\mathbf{Q}) L_{n}^{2}(\mathbf{q})}+\frac{1}{L_{n}(\mathbf{q}-\mathbf{Q}) L_{n}^{2}(\mathbf{q})}\right)\right\},
\end{aligned}
$$


Table 1 The in-plane upper critical magnetic field $H_{c 2}(\pi / 2)$

\begin{tabular}{lll}
\hline Regime & Limits of validity & $\left.H_{c 2}\left(\frac{\pi}{2}\right)\right|_{\lim }$ \\
\hline I & $H \ll \frac{\phi_{0}}{\pi d v_{F}} t$ & $\frac{m_{x} v_{F} T_{c 0}}{\hbar d t} \frac{\phi_{0}}{2 \pi}\left(1-\frac{T}{T_{c 0}}\right)$ \\
II & $\frac{\phi_{0}}{\pi d v_{F}} t \ll H \ll \frac{\phi_{0}}{\pi d v_{F}} \sqrt{t T_{c 0}}$ & $\frac{7 \zeta(3) t^{2}}{8 \pi^{3} d T_{c 0}} \sqrt{\frac{m_{x}}{T-T_{c t}}} \phi_{0}$ \\
III & $\frac{\phi_{0}}{\pi d v_{F}} \sqrt{t T_{c 0}} \ll H \ll \frac{\phi_{0}}{\pi d v_{F}} T_{c 0}$ & $\sqrt{\frac{28}{31} \frac{\zeta(3)}{\zeta(5)} \frac{T_{c t} \sqrt{T-T_{c t}}}{\hbar d t} \frac{\sqrt{2 m_{x}}}{\hbar} \phi_{0}}$ \\
IV & $H \gg \frac{\phi_{0}}{\pi d v_{F}} T_{c 0}$ & $\frac{t^{2}}{2 d v_{F}} \frac{\phi_{0}}{\left(T_{c 0}-T\right)}$ \\
\hline
\end{tabular}

where the following notation is introduced: $L_{n}(\mathbf{q})=\omega_{n}+$ $i h \operatorname{sign}\left(\omega_{n}\right)+i \frac{\mathbf{v}_{F} \mathbf{q}}{2}$. We assume that the optimum direction of the FFLO modulation vector is fixed by the symmetry of the Fermi surface and is solely determined in the paramagnetic limit $[29,30]$. The magnitude of the FFLO modulation vector is defined according to [31]. Having in our disposal the absolute value and the direction of the FFLO modulation vector, we can calculate the contribution of the orbital effect. Introducing the temperature of the Pauli limited case by $\ln \left(\frac{T_{c 0}}{T_{c P}}\right)=\pi T_{c P} \sum_{n}\left\{\frac{1}{\omega_{n}}-\left\langle\frac{1}{L_{n}(\mathbf{q})}\right\rangle\right\}$, we can write the equation for the onset of the superconductivity as

$\ln \left(\frac{T_{c P}}{T}\right)=A \pi T \sum_{n} T_{n}(\omega, \mathbf{q},+\mathbf{Q})+T_{n}(\omega, \mathbf{q},-\mathbf{Q})$,

where $T_{n}(\omega, \mathbf{q}, \pm \mathbf{Q})=\frac{t^{2}}{2} \int_{0}^{2 \pi} \frac{d \varphi}{2 \pi} L_{n}^{-1}(\mathbf{q} \pm \mathbf{Q}) L_{n}^{-2}(\mathbf{q})$, and $A$ is a function taken at $T_{C P}(H)$ and it will be displayed elsewhere [32]. This equation determines $H-T$ phase diagram for layered superconductors in the presence of the paramagnetic and the orbital effects in the limits: $t T_{c 0} \ll\left(v_{F} Q\right)^{2}$ and $t \ll T_{c 0}$.

The summation over the Matsubara frequencies of the orbital terms were performed numerically. In the sum over the Matsubara frequencies, $N=10^{4}$ terms were used. This number suffices for the convergence. The results presented in this section are in the dimensionless units. Let us start with a far-fetched situation and investigate the anisotropy of the upper critical field in the absence of the FFLO modulation vector for $H>H^{*}$. The correction to the pure Pauli limited (PL) $H-T$ phase diagram due to the orbital effects is then described by

$\ln \left(\frac{T_{c P}}{T}\right)=A \pi T t^{2} \sum_{n}\left\langle\frac{\omega_{n}\left(a^{2}+\omega_{n}^{2}-3 h^{2}\right)}{\left(\omega_{n}^{2}+h^{2}\right) z}\right\rangle$,

with $z \equiv a^{4}+2 a^{2}\left(\omega_{n}^{2}-h^{2}\right)+\left(\omega_{n}^{2}+h^{2}\right)^{2}$ and $a=\mathbf{v}_{F} \mathbf{Q} / \mathbf{2}$. At low temperature, the sum in r.h.s can become negative. However, coefficient $A$ is also negative, meaning that $T_{c P}>T$. This is seen in the polar plot in the upper panel in Fig. 1, which illustrates the magnetic field angular dependence of the normalized onset temperature in the non-modulated superconducting phase, $T_{c} / T_{c} P$, taken at $H / H_{c 0}=0.96$ and $H / H_{c 0}=0.9$, where $H_{c 0}$ is the critical magnetic field at $T=0$ in Pauli limited 2Dsuperconductors. Calculations were performed for the Fermi
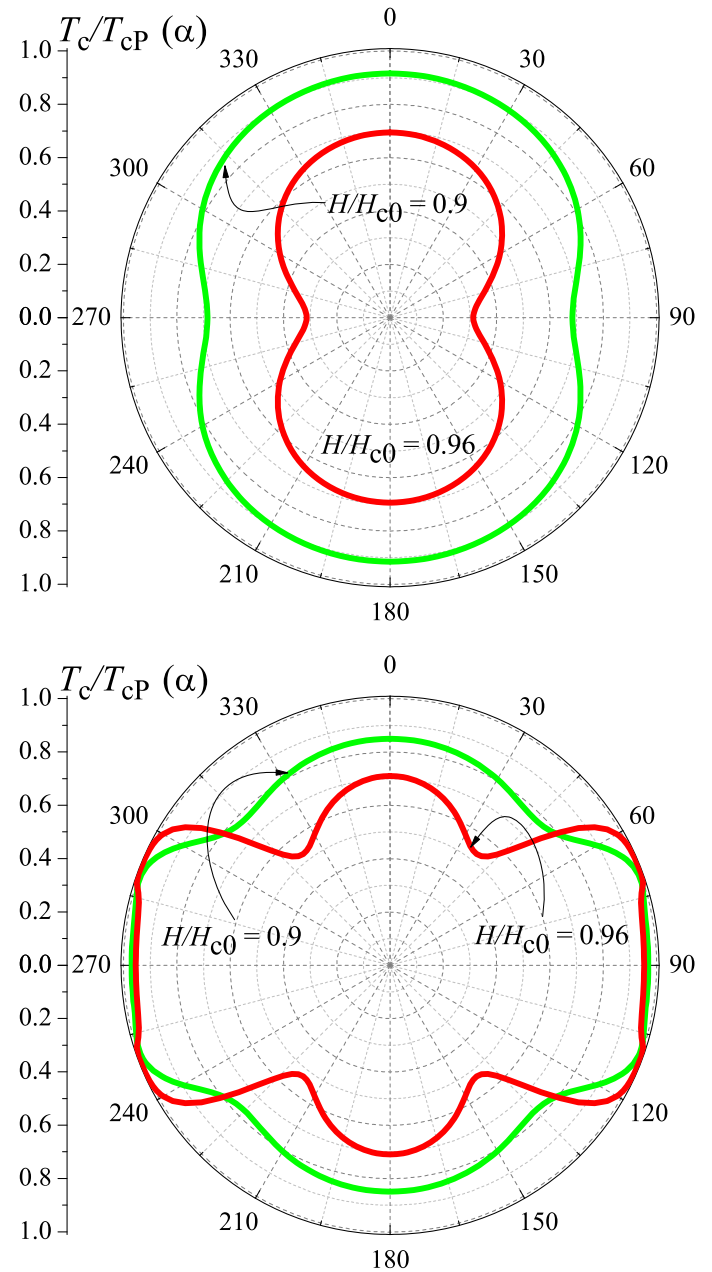

Fig. 1 The in-plane field-direction dependence of $T_{c} / T_{c P}$ at $H / H_{c 0}=0.96$ (red line) and $H / H_{c 0}=0.9$ (green line) for $m_{x}=5 m_{y}$, $q=0$ (upper panel), and $q \neq 0$ (lower panel), respectively. The Fermi velocity parameter $\eta=3.45$, and the $q$-vector is along the $x$-axis (Color figure online)

velocity $v_{F}=1.25 \times 10^{7} \mathrm{~cm} / \mathrm{s}$, or introducing dimensionless velocity parameter $\eta=\hbar v_{F} \pi d / \phi_{0} \mu_{B}$, for $\eta=3.45$, and for the in-plane mass-anisotropic situation, when $m_{x}=$ $5 m_{y}$. Hereinafter, $t / T_{c 0}=0.2$. In the polar plot, the direction of each point seen from the origin corresponds to the field direction and the distance from the origin illustrates to the normalized critical temperature. In the case of uniform 

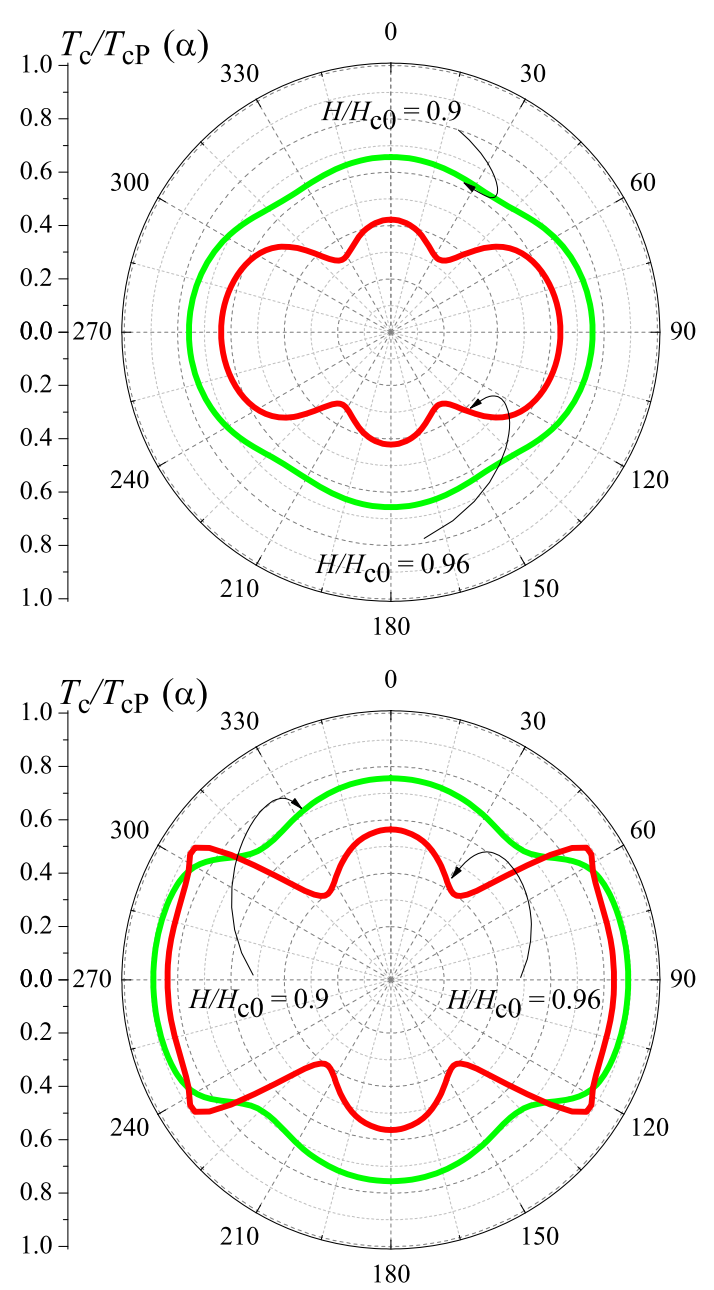

Fig. 2 The in-plane field-direction dependence of $T_{c} / T_{c P}$ at $H / H_{c 0}=0.96$ (red line) and $H / H_{c 0}=0.90$ (green line) for $m_{x}=5 m_{y}$ and $q \neq 0$. The Fermi velocity parameter $\eta$ is 0.31 (upper panel) and 0.63 (lower panel) (Color figure online)

(non-modulated) pairing, a small dip is observed at angles $\pm 90^{\circ}$ from the $x$-axis. The developments of these dips is due to the ellipsoidal Fermi line, corresponding to an ellipsoidal electronic dispersion relation. Concluding this part, one can infer that when magnetic field is rotated in the $x y$-plane, the upper critical field is smallest at angles $\pm 90^{\circ}$, i.e., when it is parallel to the direction of the lightest electron mass.

Hitherto the possibility of the formation of the FFLO state was not considered in our numerical calculations. When abating this constraint, the in-plane anisotropy of $H_{c 2}$ changes essentially. The lower panel in Fig. 1 shows the polar plot of the magnetic field direction dependence of the normalized superconducting transition temperature, taken at $H / H_{c 0}=0.96$ and $T / T_{c 0}=0.9$, for Fermi velocity parameter $\eta=3.45$ and mass anisotropy $m_{x}=5 m_{y}$. Hereinafter the $\mathbf{q}$ vector is along the $x$-axis. Comparing this result with that in the upper panel, we see that letting a pairing with the finite momentum in the layered system makes $\Delta T_{c P}$ strongly anisotropic. Namely, the position of the maximum of the onset temperature is rotated from $\alpha=0^{\circ}$, and $180^{\circ}$ typical for $q=0$, to $\alpha= \pm 70^{\circ}, \pm 110^{\circ}$ for $q \neq 0$ and $H / H_{c 0}=0.9$. The minimum in $T_{c}(\alpha)$ is rotated from $\alpha= \pm 90^{\circ}$ for $q=0$ to $\alpha= \pm 50^{\circ}, \pm 130^{\circ}$ for $q \neq 0$ and $H / H_{c 0}=0.9$.

Let us now investigate the influence of the absolute value of the Fermi velocity on the in-plane anisotropy of the upper critical field in layered superconductors. The upper and lower panels of Fig. 2 display $T_{c}(\alpha)$ for the Fermi velocity parameter $\eta=0.31$ and $\eta=0.63$, respectively. One can immediately infer that absolute value of the Fermi velocity parameter essentially influences the field-direction dependence of the superconducting onset temperature. It is seen that the position of the maximum value of $T_{c}(\alpha)$ in the case of the smallest considered Fermi velocity parameter is shifted back towards $\alpha= \pm 90^{\circ}$. The same tendency is observed for $\eta>4$ (not shown here).

In conclusion, in this work we have analyzed the fieldamplitude and the field-direction dependence of the onset temperature of the superconductivity in layered conductors. In such superconductors, the interplay of the system dimensionality, orbital pair-breaking, Pauli paramagnetism, and a possible formation of the FFLO state can lead to an anomalous in-plane anisotropy of the upper critical field.

Acknowledgements We acknowledge the support by the European Community under a Marie Curie IEF Action (Grant Agreement No. PIEF-GA-2009-235486-ScQSR), the French Project "SINUS" ANR09-BLAN-0146, and thank A. S. Mel'nikov for fruitful discussions.

\section{References}

1. Buzdin, A.I., Bulaevskii, L.N.: Sov. Phys. Usp. 27, 830 (1984)

2. Smith, R.A., Ambegaokar, V.: Phys. Rev. Lett. 77, 4962 (1996)

3. Bianconi, A., et al.: Solid State Commun. 102, 369 (1997)

4. Croitoru, M.D., et al.: Phys. Rev. B 76, 024511 (2007)

5. Shanenko, A.A., et al.: Phys. Rev. B 78, 024505 (2008)

6. Bose, S., et al.: Nat. Mater. 9, 550 (2010)

7. Croitoru, M.D., et al.: Phys. Rev. B 83, 214509 (2011)

8. Croitoru, M.D., et al.: Phys. Rev. B 84, 214518 (2011)

9. Lebed, A.G. (ed.): The Physics of Organic Superconductors and Conductors. Springer, Berlin (2008)

10. Lee, I.J., et al.: Phys. Rev. Lett. 78, 3555 (1997)

11. Lee, I.J., et al.: Phys. Rev. B 62, R14669 (2000)

12. Lee, I.J., et al.: Phys. Rev. B 65, 180502(R) (2002)

13. Oh, J.I., Naughton, M.J.: Phys. Rev. Lett. 92, 067001 (2004)

14. Yonezawa, S., et al.: Phys. Rev. Lett. 100, 117002 (2008)

15. Larkin, A.I., Ovchinnikov, Yu.N.: Zh. Eksp. Teor. Fiz. 47, 1136 (1964). [Sov. Phys. JETP 20, 762 (1965)]

16. Fulde, P., Ferrell, R.A.: Phys. Rev. 135, A550 (1964)

17. Lee, I.J., et al.: Phys. Rev. Lett. 88, 017004 (2001)

18. Shinagawa, J., et al.: Phys. Rev. Lett. 98, 147002 (2007)

19. Wright, J.A., et al.: Phys. Rev. Lett. 107, 087002 (2011)

20. Lebed, A.G., Wu, S.: Phys. Rev. B 82, 172504 (2010)

21. Matsuda, Y., Shimahara, H.: J. Phys. Soc. Jpn. 76 (2007) 
22. Shimahara, H.: In: Lebed, A.G. (ed.) The Physics of Organic Superconductors and Conductors. Springer, Berlin (2008)

23. Buzdin, A.I.: Rev. Mod. Phys. 77, 935 (2005)

24. Innocenti, D., et al.: Phys. Rev. B 82, 184528 (2010)

25. Kopnin, N.B.: Theory of Nonequilibrium Superconductivity. Clarendon, Oxford (2001)

26. Lebed, A.G.: Pis'ma Zh. Eksp. Teor. Fiz. 44, 89 (1986). [JETP Lett. 44, 114 (1986)]

27. Burlachkov, L.I., et al.: Europhys. Lett. 4, 941 (1987)
28. Saint-James, D., Sarma, G., Thomas, E.J.: Type II Superconductivity. Pergamon, Oxford (1969)

29. Buzdin, A., Matsuda, Y., Shibauchi, T.: Europhys. Lett. 80, 67004 (2007)

30. Denisov, D., Buzdin, A., Shimahara, H.: Phys. Rev. B 79, 064506 (2009)

31. Bulaevskii, L.N.: Zh. Eksp. Teor. Fiz. 65, 1278 (1973). [Sov. Phys. JETP 38, 634 (1974)]

32. Croitoru, M.D., et al.: Phys. Rev. Lett. (in press) 